\title{
CONCEPÇÕES SOBRE BEM-ESTAR FÍSICO DE ESCOLARES DOS ANOS FINAIS DO ENSINO FUNDAMENTAL
}

\section{CONCEPTIONS OF PHYSICAL WELLNESS OF SCHOOL OF FINAL YEARS OF ELEMENTARY EDUCATION}

\section{Liliani Mathias Brum¹, Cati Reckelberg Azambuja², Cristiane Köhler Carpilovsky ${ }^{3}$, Maria Rosa Chitolina Schetinger ${ }^{4}$}

\footnotetext{
${ }^{1}$ PPG Educação em Ciência: Química da Vida e Saúde, UFSM, lilianibrum@gmail.com ${ }^{2}$ PPG Educação em Ciência: Química da Vida e Saúde, UFRGS, cati.razambuja@ hotmail.com

${ }^{3}$ Departamento de Morfologia, UFSM, criskoh@terra.com.br

${ }^{4}$ Departamento de Química e PPG Educação em Ciências: Química da Vida e Saúde, UFSM, mariachitolina@gmail.com
}

\section{RESUMO}

O ensino de ciências tem sido um desafio para a educação. O objetivo deste estudo foi avaliar as concepções sobre bem-estar físico dos alunos do ensino fundamental. $\mathrm{O}$ instrumento utilizado foi um questionário semi-estruturado submetido à Análise de Conteúdo e Estatística Descritiva. Como resultado, 56,9\% (n=87), dos entrevistados consideraram que bem-estar físico está relacionado à saúde do organismo e 64,7\% $(\mathrm{n}=99)$ responderam sentir-se melhor através da atividade física. Conclui-se que a maioria dos alunos considera que bem-estar físico está relacionado à saúde do organismo e acreditam que por meio da prática de atividade física se sentirão melhor com o seu corpo.

Palavras-chave: Estudantes, Saúde Escolar, Atividade Física.

\begin{abstract}
Science teaching has been a challenge for education. The objective of this study was to estimate the conceptions about physical well-being of Fundamental Education students. The instrument utilized was a semi-structured questionnaire submitted to the Analysis of Content and Descriptive Statistics. As a result, 56.9\% ( $\mathrm{n}=87)$, of the interviewed considered that physical well-being is related to the health organism, and $64.7 \%(n=99)$ answered that they felt better with physical activity. One concludes that most of the students consider that physical welfare is related to organism health and they believe that through physical activity practice they will feel better with their body.
\end{abstract}

Keywords: Students, School Health, Physical Activity.

\section{Introdução}

A adolescência é compreendida pela maior parte dos estudiosos do desenvolvimento humano como um período de mudanças físicas, cognitivas e sociais que, juntas, ajudam a traçar o perfil desta população (FROTA, 2007). É na adolescência que, sob diversos ângulos, vemos conceitos e atitudes serem substituídos: são novas 
maneiras de se pensar, de se relacionar, de se comunicar e de se movimentar. Esses indícios sinalizam para o amadurecimento do ser humano e para a sua inserção na vida adulta (PIAGET, 1998).

A adolescência seria uma fase do desenvolvimento humano que faz uma ponte entre a infância e a idade adulta, um período atravessado por crises, que encaminham o jovem na construção de sua subjetividade. Neste sentido, Samdal et al. (1998) sugeriram que a percepção que os alunos têm da vida escolar influencia a sua percepção de bemestar e adoção de comportamentos ligados á saúde.

O ensino de ciências, orientado pelos Parâmetros Curriculares Nacionais (PCNs), deve buscar situações relevantes na vivência dos estudantes e tematizá-las através da proposição de eixos transversais ao currículo da disciplina (BRASIL, 1998). Assim, Nutbeam et al. (1993) defendem que a educação para a saúde tradicional nas escolas é inadequada.

A educação apresenta o ensino de ciências como um desafio à medida que o aluno deve ser estimulado a questionar e pensar acerca dos temas propostos na aprendizagem e relevantes no seu papel de cidadão. A saúde é um destes temas e tem como objetivo articular-se com o contexto sócio-cultural e com o mundo que circunda o aluno (FREITAS e MARTINS, 2008).

Estabelecer os conhecimentos e competências a serem adquiridos pelos alunos a respeito da saúde engloba a abordagem da qualidade de vida. O conceito de qualidade de vida, segundo Barros et al. (2008), é multidimensional, que inclui bem-estar (material, físico, social, emocional e produtivo) e satisfação em várias áreas da vida. A satisfação com a vida ou a apreciação subjetiva da qualidade devida, tem sido considerada como o julgamento que o indivíduo faz sobre diferentes áreas da vida, sendo os critérios mais utilizados nestas análises os desejos e expectativas, assim como comparações com um grupo de referências e experiências prévias.

Gaspar et al. (2006), relatam que a qualidade de vida e o bem-estar devem ser considerados sob uma perspectiva ecológica que foca múltiplos níveis de análise, a criança, os pais e a família, os pares, a comunidade e a sociedade, quando relacionados com a saúde da criança e do adolescente. Adolescentes pobres apresentam maior risco para a saúde e para o bem-estar em nível físico, mental e social, pois estão expostas com maior frequência a doenças físicas, estresse familiar, suporte social insuficiente e depressão parental, especialmente da mãe. 


\section{Adolescência e Saúde}

A adolescência é uma categoria sociocultural, historicamente construída a partir de critérios múltiplos que abrangem tanto a dimensão bio-psicológica, quanto à cronológica e a social. O fato é que estar na adolescência é viver uma fase em que múltiplas mudanças acontecem e se refletem no corpo físico, pois o crescimento somático e o desenvolvimento em termos de habilidades psico-motoras se intensificam e os hormônios atuam vigorosamente levando a mudanças radicais de forma e expressão (FERREIRA et al., 2007)

No século XX, diante destes avanços, a definição de saúde ultrapassou a barreira da simples ausência de doenças. Ela está identificada com diversos aspectos do comportamento humano que estão em interação com um completo bem-estar físico, mental e social. Todas as dimensões que compõem a saúde podem transitar por pólos positivos e negativos, ou seja, a saúde positiva está associada com a capacidade de apreciar a vida e de resistir aos desafios do cotidiano, enquanto a saúde negativa está vinculada à morbidade e, até mesmo, à mortalidade (PITANGA, 2002).

No campo do cuidado á saúde, em especial do adolescente, ações educativas vêm demonstrando possibilidade de convergência bastante eficaz entre os dois campos: do cuidado e da educação. Segundo Ferreira et al. (2007), tais ações, voltadas aos adolescentes e pensadas á luz da educação, devem valorizar os saberes dos jovens na construção de uma proposta de atenção básica e centrada no sujeito do cuidado.

Ainda mais, a aliança entre metodologias de pesquisa participativas e a educação popular em saúde tem sido debatida como uma estratégia profícua no campo da atenção básica porque promove o diálogo intersubjetivo e a interação entre sujeitos e pesquisadores. Tal estratégia vem demonstrando possibilidade de convergência bastante eficaz entre os dois campos: do cuidado e da educação.

A Organização Mundial de Saúde (OMS) define saúde não apenas como a ausência de doença, mas como a situação de perfeito bem-estar físico, mental e social. No campo da saúde, o discurso da relação entre saúde e qualidade de vida existe desde o nascimento da medicina social, nos séculos XVIII e XIX, quando investigações sistemáticas começaram a referendar esta tese e oferecer subsídios para as políticas públicas. Quanto mais aprimorada a democracia, mais ampla é a noção de qualidade de vida, o grau de bem-estar da sociedade e de igual acesso a bens materiais e culturais (MATOS, 1998). Assim, em determinado tempo de seu desenvolvimento econômico, 


\section{Ensino, Saúde e Ambiente - V5 (3), pp. 55-68, dez. 2012}

social e tecnológico, uma sociedade específica tem um parâmetro de qualidade de vida diferente da mesma sociedade em outra etapa histórica (MINAYO et al., 2000).

\section{Educação e Saúde}

Para Focesi (1990), a maior responsabilidade do processo de educação em saúde é a do professor, cabendo a este colaborar para o desenvolvimento do pensamento crítico do escolar, além de contribuir para que as crianças adotem comportamentos favoráveis à saúde. Os docentes da educação fundamental desempenham um importante papel neste contexto, por estarem atuando diretamente com crianças em processo de formação intelectual e desenvolvimento de condutas.

Durante a infância, época decisiva na construção de hábitos e atitudes, a escola assume um papel importante por seu potencial para o desenvolvimento de um trabalho sistematizado e contínuo. Os valores que se expressam na escola em seus diferentes aspectos geralmente são aprendidos pelas crianças na sua vivência diária (FERNANDES et al., 2005).

Diante dessas perspectivas, o Ministério da Educação e do Desporto (BRASIL, 1998), criou o referencial curricular nacional para a educação fundamental, no qual a saúde é tida como um tema transversal a ser trabalhado e assumido com responsabilidade no projeto de toda a escola; alunos, professores e o ambiente escolar tornam-se sistematicamente elementos chaves para essa realização.

Apesar das normas existentes, o professor em suas práticas diárias não vem cumprindo de maneira eficaz o que está previsto nos PCNs, o que se pode atribuir ao processo de formação docente. As escolas também não se sentem responsáveis pela prática da saúde em seus ambientes e geralmente reproduzem o paradigma de caráter assistencialista, priorizando o indivíduo e a doença, em detrimento da coletividade e da prevenção (FERNANDES et al., 2005).

Oliveira (1997) ressalta que os docentes geralmente não conhecem as características do desenvolvimento dos escolares, o que pode dificultar o trabalho de questões ligadas à saúde. Conceição (1994), em seus estudos, também conclui que os professores não são adequadamente preparados para trabalhar com o ensino de saúde, ideia corroborada por Oliveira e Lopes Silva (1990), os quais caracterizam o processo de formação dos docentes como falho nessa área, geralmente centrado nas práticas pedagógicas transmissoras de informações desvinculadas da realidade. 
A escola saudável deve ser entendida como um espaço vital gerador de autonomia, participação, crítica e criatividade, para que o escolar tenha a possibilidade de desenvolver suas potencialidades físicas, psíquicas, cognitivas e sociais (PELICIONI, 1999).

Enquanto fator de promoção de saúde e parâmetro indicador de qualidade de vida, o estilo de vida tornou-se fundamental na ação preventiva a doenças. Acontece principalmente na medida em que a pessoa passa a ser responsável pela administração da sua saúde e do bem-estar, o que reflete diretamente no equilíbrio no meio em que o cerca (MINAYO et al., 2000).

Além do estilo de vida, alguns itens ligados às condições de vida, tais como o acesso aos sistemas de saúde e à escolaridade, a exposição aos riscos ocupacionais e ambientais e as oportunidades de trabalho também influenciam o modus vivendi de uma sociedade (MINAYO et al., 2000; LESSA, 1999). Segundo Candeias (1997), a expressão condições de vida, permite que o conceito em promoção de saúde vença os limites dos fatores comportamentais para prender-se em uma rede de interação mais complexa, constituída pela cultura, por normas e pelo ambiente sócio-econômico. Inclusive consta da Lei Federal 8.080 de 1990 (BRASIL, 1990) em seu Artigo 3º, a proposição de que a saúde possui um conjunto de fatores determinantes e condicionantes caracterizados pela alimentação, moradia, saneamento básico, transporte, meio-ambiente, trabalho, renda, educação, lazer e serviços essenciais.

Diante da relevância da temática do eixo transversal - saúde e, do público alvo os adolescentes, este estudo teve por objetivo investigar as concepções de bem-estar físico dos alunos do ensino fundamental em uma escola pública no interior do Rio Grande do Sul.

\section{Metodologia}

A pesquisa, de caráter descritivo (GIL, 1991), teve sua população constituída por alunos do ensino fundamental de uma escola pública estadual do município de São Sepé, RS. A amostra total foi de 198 alunos matriculados regularmente nas séries finais do ensino fundamental (EF).

A aprovação pelo Comitê de Ética em Pesquisa com Humanos, sob o número 0202.0.243.000-09 - CAAE permitiu o inicio do trabalho de campo com as visitas à instituição de ensino que oferece ensino fundamental e médio, em três turnos. 
O projeto de pesquisa foi apresentado à direção da escola e após a autorização da mesma, a coleta de dados foi realizada no período de compreendeu os meses de abril a julho de 2010, nos turnos da manhã e tarde.

Para esta coleta, utilizou-se um questionário, semi-estruturado e a observação participante (GOLDENBERG, 2005; GIL, 1999; TRIVIÑOS, 1994). Além disso, os dados receberam os tratamentos de Análise de Conteúdo (BARDIN, 2004; MORAES, 1999) e Estatística Descritiva (REIS, 1998).

A observação participante iniciou com a primeira visita á escola e estendeu-se por todo período em que foram realizadas as atividades, pois conforme ressalva (TRIVIÑOS, 1994), em pesquisa social a observação é constante. Para a Análise de Conteúdo seguiram-se alguns passos preconizados pela literatura pertinente quais sejam: a) leitura flutuante; b) desconstrução e unitarização dos textos; c) categorização; d) construção de um metatexto com expressão das compreensões atingidas.

O questionário utilizado no estudo foi composto por perguntas de caráter sóciodemográfico, de saúde, qualidade de vida e bem-estar. As questões que se referiram à saúde, qualidade de vida e bem-estar foram elaboradas no formato "perguntas abertas" que, segundo Gil (1999), tem a vantagem de não forçar o respondente a enquadrar a sua percepção em alternativas preestabelecidas. O instrumento também foi submetido à Validação através da análise de sua Matriz Analítica por três professores da área, que sugeriram correções, as quais foram acatadas.

Além das questões de caráter sócio-demográfico como idade, sexo e ano escolar, os alunos também responderam as seguintes perguntas: 1) Quantas vezes você já esteve afastado da escola por problemas de saúde? 2) Quantas vezes você já esteve hospitalizado? 3) Você utiliza remédios diariamente (quais)? 4) Quantas vezes por semana você realiza atividades físicas? 5) Quantas vezes por mês você realiza atividades de lazer (quais)? 6) O que você considera bem-estar físico? 7) O que você acha que poderia fazer para se sentir melhor com o seu corpo?

A grande variedade de respostas fornecidas pelos estudantes, ao responder o instrumento de pesquisa, foi submetida à análise de conteúdo para a categorização por temáticas, onde surgiram as seguintes categorias nominadas de: 1) Atividade Física para a prática de exercícios físicos de forma sistemática, como por exemplo, jogar, praticar futebol, ginástica, praticar voleibol, andar de bicicleta; 2) Saúde e Organismo para designar condições de saúde, doenças, órgãos e alimentação saudável; 3) Lazer para 
respostas que indicavam bem-estar, sair para passear, relaxar e festas; 4) Cultura para as relações de meio ambiente, trabalho e com os pais; 5) Positivo para as respostas que referiram-se de forma positiva, como por exemplo, "sim" e "tudo"; 6) Negativo para aquelas que afirmaram o "não" de forma categórica; 7) Não respondeu para os que não responderam aos questionamentos ou que afirmaram não estar de acordo com o objetivo da questão, "nada" ou "não sei”.

\section{Resultados e Discussão}

A pesquisa foi realizada com os alunos do $6^{\circ}$ ao $9^{\circ}$ ano do EF de uma escola pública localizada em uma cidade da região central do estado do Rio Grande do Sul. Os alunos das séries finais do EF foram convidados a responder o questionário e, dos 198 alunos matriculados regularmente, 77,3\% (n=153) consentiram em participar do estudo, sendo 77 do sexo feminino e 76 do sexo masculino. A caracterização da amostra, estratificada por sexo e ano escolar, está apresentada na Tabela 1.

Tabela 1 - Distribuição dos alunos do Ensino Fundamental, estratificados por sexo e ano escolar.

\begin{tabular}{|c|c|c|c|c|c|c|}
\hline \multirow{3}{*}{$\begin{array}{c}\text { Ano Escolar } \\
\text { Ensino Fundamental }\end{array}$} & \multirow{2}{*}{\multicolumn{2}{|c|}{ N Total }} & \multicolumn{4}{|c|}{ Sexo } \\
\hline & & & \multicolumn{2}{|c|}{ Feminino } & \multicolumn{2}{|c|}{ Masculino } \\
\hline & $\mathbf{N}$ & $\%$ & $\mathbf{N}$ & $\%$ & $\mathbf{N}$ & $\%$ \\
\hline $6^{\circ}$ Ano & 50 & 32,7 & 29 & 18,9 & 21 & 13,7 \\
\hline $7^{\circ}$ Ano & 35 & 22,9 & 13 & 8,5 & 22 & 14,4 \\
\hline $8^{o}$ Ano & 36 & 23,5 & 14 & 9,2 & 22 & 14,4 \\
\hline $9^{\circ}$ Ano & 32 & 20,9 & 21 & 13,7 & 11 & 7,2 \\
\hline Total & 153 & 100,0 & 77 & 50,3 & 76 & 49,7 \\
\hline
\end{tabular}

Segundo Carvalho (1997), é nas séries do ensino fundamental que encontra-se a grande maioria da população estudantil brasileira, sendo este nível de ensino responsável pelos primeiros contatos dos alunos com determinados conceitos científicos em uma situação de ensino, e que serão extremamente importantes para a aprendizagem subsequente em Ciências.

A idade média dos alunos pesquisados, em anos, foi de $11,8 \pm 1,0$ para o $6^{\circ}$ ano, $12,7 \pm 1,1$ para o $7^{\circ}$ ano, $14,1 \pm 1,5$ para o $8^{\circ}$ ano e $14,9 \pm 1,9$ para o $9^{\circ}$ ano. A variação das idades mínimas e máximas tornou-se proporcionalmente maior à medida que 
progrediu o ano escolar: de 10 à 13 anos ( $6^{\circ}$ ano), de 11 à 16 anos $\left(7^{\circ}\right.$ ano $)$, de 12 à 20 anos ( $8^{\circ}$ ano) e 13 à 23 anos ( $\left(9^{\circ}\right.$ ano).

$\mathrm{Na}$ caracterização da amostra, no que diz respeito a algumas questões de saúde, $50,3 \%(n=77)$ dos alunos responderam que já ficaram afastados entre uma e dez vezes da escola por problemas de saúde, 57,5\% (n=88) afirmaram não ter sido hospitalizado nenhuma vez até o momento da pesquisa e 86,3\% $(n=132)$ disseram não usar remédios diariamente. Entre aqueles que relataram uso diário de medicamentos, a maior incidência foi para a administração de analgésicos.

O importante determinante de uma boa saúde, a prática de atividades físicas, foi mencionada com as frequências de cinco vezes ou mais por semana $(36,6 \%$; $=56)$, de uma a quatro vezes na semana $(58,2 \% ; n=89)$ e nenhuma vez na semana $(5,2 \% ; n=8)$. As atividades de lazer foram citadas, na sua grande maioria $(71,2 \% ; n=109)$, como realizadas entre uma e dez vezes por mês. Os exemplos mais frequentes encontrados nas respostas dos alunos foram: "ir á praça central, passear em casas de parentes, ir ao clube, viajar, ir ao sitio, ir ao parque de diversões e visitar amigos".

Muitos dos valores e atitudes são formados e adquiridos durante a adolescência, principalmente aqueles que dizem respeito à adoção de hábitos saudáveis de vida e prática regular de atividades físicas (ROLIM et al., 2007). Estudos como os de Matias et al. (2009) e Nahas (2003), têm demonstrado que a atividade física possui um forte impacto nos diversos domínios que compõem o estilo de vida. Segundo Pires et al. (2004), a prática de atividades físicas regulares contribui para redução da pressão arterial e da massa corporal, além de beneficiar a quantidade de conteúdo mineral e densidade óssea dos adolescentes.

Do total de alunos do EF participantes deste estudo, 56,9\% $(n=87)$, consideraram que bem-estar físico está relacionado à saúde do organismo de forma geral, enquanto que $23,5 \%(\mathrm{n}=36)$ à atividade física, $15,7 \% \quad(\mathrm{n}=24)$ ao lazer e, 3,9\% (n=6) não responderam a esta pergunta.

A visão que os adolescentes tem de bem-estar está relacionada com a possibilidade destes integrarem comportamentos de saúde no seu estilo de vida (NUTBEAM et al., 1993). Segundo os alunos que participaram desta pesquisa, a ausência de doenças, bom funcionamento dos órgãos e a manutenção de uma alimentação saudável, caracterizaram a percepção sobre o bem-estar físico da maioria dos alunos que responderam os questionários. Esta visão está em conformidade com os 
86,3\% (n=132) dos entrevistados que disseram não usar remédios diariamente. Da mesma forma, o fato de 57,5\% ( $\mathrm{n}=88)$ afirmarem não ter sido hospitalizado nenhuma vez até o momento da pesquisa, demonstra que existe uma coerência entre o "real" e o "ideal".

Contudo, como mais da metade dos alunos afirmaram já terem ficado afastados alguma vez da escola por problemas de saúde, pressupõe que pequenos problemas de saúde, não tão graves, podem interferir no rendimento individual, no momento em que afastam os alunos dos bancos escolares. Além disso, apesar de não ter sido maioria, outro problema aparece nas entre-linhas da pesquisa - o abuso de medicação - relatada por $13,7 \%(n=21)$ dos alunos que utilizam medicamentos diariamente, sendo a maior incidência, a classe de analgésicos.

O bem-estar e a qualidade de vida relacionada com a saúde da criança e do adolescente devem ser considerados sob múltiplos níveis de análise, a criança, os pais e a família, os pares, a comunidade e a sociedade (HARDING, 2001; NELSON et al., 2001). A resposta da maioria dos alunos vai ao encontro do que preconizam os autores.

Matos e Carvalhosa (2001) dizem que a mais relevante influência na percepção que os jovens têm do seu bem-estar é a sua percepção de um ambiente positivo na escola. Esta ideia nos mostra o quanto se torna importante o papel da escola para a vida dos adolescentes, transpondo as fronteiras do conhecimento formal e influenciando a percepção que estes têm do seu bem-estar.

Os objetivos da educação para a saúde, na escola, são os de formar uma personalidade autônoma, capaz de construir seu próprio estilo de vida e conseguir um equilíbrio que lhe proporcione bem-estar no terreno físico, psíquico e social; oferecer os meios para que o aluno se conscientize de seus próprios estados físicos e psíquicos, dos hábitos e atitudes diante das diversas situações da vida cotidiana (BUSQUETS et al., 1998).

O estilo de vida e o nível habitual de atividade física de adolescentes têm sido positivamente correlacionados pela literatura. Pressupõe-se, de acordo com as pesquisas, que o indivíduo terá mais saúde e bem-estar, quanto mais ele equilibrar seu estilo de vida: realizar atividade física regular, alimentar-se adequadamente e com qualidade, controlar o estresse, manter relacionamentos positivos e estáveis e comportamentos preventivos como o não uso de drogas, evitar o tabagismo e fazer sexo com camisinha (NAHAS, 2003). 
Além disso, estudos epidemiológicos e documentos institucionais propõem que a prática regular de atividade física e uma maior aptidão física estão associadas a uma menor mortalidade e melhor qualidade de vida em população adulta. Não são poucos os trabalhos científicos que destacam o sedentarismo e o estresse como responsáveis por doenças hipocinéticas e reduções na qualidade de vida. Existem cada vez mais dados demonstrando que o exercício, a aptidão e a atividade física estão relacionados com a prevenção, com a reabilitação de doenças e com a qualidade de vida. Em adendo, um corpo crescente na literatura demonstra que a participação em diferentes formas de atividade física é associada positivamente com a saúde mental (ARAÚJO e ARAÚJO, 2000).

Quando os alunos foram questionados a respeito do que eles poderiam fazer para se sentir melhor com o seu corpo, 64,7\% (n=99) responderam que poderiam alcançar este objetivo através da atividade física e 18,3\% (n=30) recorreram a respostas ligadas à saúde e organismo. Um alto percentual $(17,0 \% ; n=26)$ não responderam a questão ou não souberam dizer o que poderiam fazer.

No estudo que realizamos, foi observado que 94,8\% $(\mathrm{n}=145)$ praticam algum tipo de atividade física, pelo menos uma vez na semana. Hohepa e cols. (2006), observaram que os adolescentes relacionam os efeitos benéficos da prática da atividade física a alegria, a realização e a benefícios físicos e psicológicos. A atividade física na adolescência pode estimular o crescimento físico, aumentar a auto-estima, contribuir para o desenvolvimento social, além de propiciar uma série de benefícios para a saúde e bem-estar (VIEIRA et al., 2002).

Estudos têm indicado que pessoas mais ativas fisicamente apresentam melhores escores de qualidade de vida (SGUIZZATTO et al., 2006). Avaliando 461 adolescentes do sexo masculino, entre 17 e 19 anos, que estavam se apresentando na Junta de Alistamento Militar do Ibirapuera em São Paulo, Alves et al. (2000), encontraram 81\% referindo prática atual ou pregressa de algum tipo de atividade física, independentemente da educação física compulsória nas escolas. Os autores verificaram ainda, que $42,5 \%$ dos adolescentes começaram a desenvolver algum tipo de atividade física antes dos 12 anos de idade, $51 \%$ entre 12 e 16 anos e 6,5\% após os 16 anos. Além disso, $47 \%$ mencionaram interrupção na prática de atividade física, sendo que esta ocorreu predominantemente após os 15 anos. 
Segundo Barros (1993), os adolescentes praticam exercícios por várias razões, que se diferenciam com a idade. Os principais fatores podem ser assim agrupados: para adquirir autoconfiança e satisfação pessoal, para sair da rotina das atividades curriculares, para se sociabilizar e para simular objetivos de vida, já que o esporte pode ser um palco de situações a serem vivenciadas na idade adulta. Segundo Priore (1998), a grande preocupação com a imagem corporal geralmente é o estímulo que leva muitos adolescentes a buscar atividades desportivas, sobretudo extracurriculares.

Gambardela (1995) estudou 273 adolescentes estudantes do período noturno, verificando que a prática esportiva era mais prevalente entre os indivíduos do sexo masculino e, destes, entre os que trabalhavam, sendo que estes últimos relataram dedicar de 9 a 15 horas por semana a esta atividade.

\section{Considerações Finais}

O papel da escola frente ao desenvolvimento dos temas transversais, como a educação para a saúde, possui objetivos de formação de personalidade e estilo de vida que proporcione aos escolares, capacidades e autonomia para suas decisões em relação ao seu bem-estar físico, psíquico e social.

Importante constatação, foi a de que a percepção de bem-estar físico, para os alunos entrevistados, parece ser fortemente determinada pela a ausência de doenças, bom funcionamento dos órgãos e pela manutenção de uma alimentação saudável. Para a maioria dos alunos, a prática de atividades físicas proporciona benefícios para o organismo, pois acreditam que por meio da realização destas sentir-se-iam melhor em relação ao seu corpo.

Compreende-se assim, que a saúde e a educação são inseparáveis e interdependentes, pois, para se ter uma, necessita-se da outra. Ao mesmo tempo em que a saúde só é alcançável quando se tem uma boa educação. Em fim, ressalta-se que a educação e a saúde são necessidades sociais, que devem ser garantidas pelas instituições governamentais.

\section{Referências Bibliográficas}

ARAÚJO, D.S.M.S.; ARAÚJO, C.G.S. Aptidão física, saúde e qualidade de vida relacionada à saúde em adultos. Revista Brasileira de Medicina do Esporte [online], v. 6, n. 5, p. 194-203, 2000. 
ALVES, S.S.; SILVA, S.R.C.; RIBEIRO, R.S.; VERTEMATTI, A.S.; FISBERG, M. Avaliação de atividade física, estado nutricional e condição social em adolescentes.

Folha Médica, n. 119, p. 26-33, 2000.

BARDIN, L. Análise de Conteúdo. $3^{\text {a }}$ ed. Lisboa: Edições 70, 2004.

BARROS, L.P.; GROPO, L.N.; PETRIBÚ, K.; COLARES, V. Avaliação da qualidade de vida em adolescentes - revisão da literatura. Jornal Brasileiro de Psiquiatria, v. 57, n. 3, p. 212-217, 2008.

BARROS, R. Os adolescentes e o tempo livre: lazer - atividade física. In: Coates Verônica; Françoso, Lucimar Aparecida; Beznos, Geni Worcman. Medicina do adolescente. São Paulo: Sarvier, 1993.

BRASIL. Ministério da Saúde. Lei no 8.080, 19 de Setembro de 1990. Dispõe sobre as condições para a promoção, proteção e recuperação da saúde, a organização e o funcionamento dos serviços correspondentes e dá outras providências. Disponível em <http://portal.saude.gov.br/portal/arquivos/pdf/LEI8080.pdf> Acesso em: 10 maio 2012.

Secretaria de Educação Fundamental. Parâmetros Curriculares Nacionais, Brasília: MEC/SEF, v. 4, 1998.

BUSQUETS, M.D.; LEAL, A. A educação para a saúde. In: BUSQUETS M.D.; CANDEIAS, N.M.F. Conceitos de educação e de promoção em saúde: mudanças individuais e mudanças organizacionais. Revista de Saúde Pública, v. 31, n. 2, p. 20913, 1997.

CARVALHO, A.M.P. Ciências no Ensino Fundamental. Cadernos de Pesquisa, n. 101, p.152-68, 1997.

CONCEIÇÃO, J. A. N. Conceito de saúde escolar. In: Saúde escolar: a criança, a vida e a escola. São Paulo: Savier, 1994.

FERREIRA, M.A.; ALVIM, N.A.T.; TEIXEIRA, M.L.O.; VELOSO, R.C. Saberes de adolescentes: estilo de vida e cuidado à saúde. Texto Contexto - Enfermagem, v. 16, n.2, p. 217-24, 2007.

FERNANDES, M.H.; ROCHA, V.M.; SOUZA, D.B. A concepção sobre saúde do escolar entre professores do ensino fundamental ( $1^{\mathrm{a}} \mathrm{a} 4^{\mathrm{a}}$ séries). História, Ciências, Saúde - Manguinhos, v. 12, n 2, p. 283-91, 2005.

FOCESI, E. Formação em saúde na escola. O papel do professor. Revista Brasileira Saúde do Escolar, v. 1, n. 2, p. 4-8, 1990.

FREITAS, E.O.; MARTINS, I. Concepções de saúde no livro didático de ciências.

Ensaio - Pesquisa em Educação em Ciências, v. 10, n.2, 2008.

FROTA, A.M.M.C. Diferentes concepções da infância e adolescência: a importância da historicidade para sua construção. Estudos e pesquisas em psicologia, v. 7, n. 1, p. 147-60, 2007.

GAMB ARDELLA, A.M.D. Adolescentes estudantes de período noturno: como se alimentam e como gastam suas energias. 1995, (Tese de Doutorado / Universidade de São Paulo), São Paulo, 1995.

GASPAR, T.; MATOS, M.G.; RIBEIRO, J.L.P.; LEAL, I. Qualidade de vida e bemestar em crianças e adolescentes. Revista Brasileira de Terapias Cognitivas, v. 2, n. 2, 2006. Disponível em:

http://pepsic.bvsalud.org/scielo.php?script=sci arttext\&pid=S1808-

56872006000200005\&lng=pt\&nrm=iso. Acesso em 05 jun. 2012.

GIL, A.C. Como elaborar projetos de pesquisa. São Paulo: Atlas, 1991. Métodos e Técnicas de Pesquisa Social. São Paulo: Atlas, 1999. 
GOLDENBERG M. A arte de pesquisar - como fazer pesquisa qualitativa em Ciências Sociais, $9^{\mathrm{a}}$ ed. Rio de Janeiro - São Paulo: Record, 2005.

HARDING, L. Children's quality of life assessments: a review of genetic and health related quality of life measures completed by children and adolescents. Clinical Psychology and Psychotherapy, n. 8, p. 79-96, 2001.

HOHEPA, M.; SCHOFIELD, G.; KOLT, G. S. Physical activity. What do high school students think? Journal of Adolescent Health, 39(3):328-36, 2006.

LESSA, I. Doenças crônico não-transmissíveis: bases epidemiológicas. In:

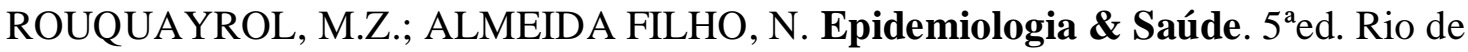
Janeiro: Medsi, 1999.

MATIAS, T. S.; ROLIM, M. K. S. B.; KRETZER, F. L.; SCHMOELZ, C. P.;

VASCONCELLOS, D. I. C.; ANDRADE, A. Estilo de vida, nível habitual de atividade física e percepção de autoeficácia de adolescentes. Revista da Educação Física/UEM, 20(2):235-43, 2009

MATOS, M. G.; CARVALHOSA, S. F. A Saúde dos adolescentes: ambiente escolar e bem-estar. Psicologia, Saúde \& Doenças, 2(2):43-53, 2001.

MATOS, O. As formas modernas do atraso. Folha de São Paulo, Primeiro Caderno, 27 de setembro, p. 3, 1998.

MINAYO, M.C.S.; HARTZ, Z.M.A.; BUSS, P.M. Qualidade de vida e saúde: um debate necessário. Ciência \& Saúde Coletiva, v. 5, n. 1, p. 7-18, 2000.

MORAES, R. Análise de conteúdo. Revista Educação, v. 22, n. 37, p. 7-32, 1999.

NAHAS, M.V. Atividade física, saúde e qualidade de vida: conceitos e sugestões para um estilo de vida ativo. $3^{a}$ ed. Londrina: Midiograf, 2003, 278p.

NELSON, G.; LAURENDEAU, M.C.; CHAMBERLAND, C. A review of programs to promote family wellness and prevent the maltreatment of children. Canadian Journal of Behavioural Science, v. 33, n. 1, p. 1-13, 2001.

NUTBEAM, D. SMITH, C.T.; MOORE, L.; BAUMAN, A. Warning! School can damage your health: Alienation from school and its impact on health behaviour.

Journal of Pediatric Child Health, v. 29 (suppl.), p. 825-30, 1993.

OLIVEIRA, M. L. C. Concepções, dificuldades e desafios nas ações educativas em saúde para escolares no Brasil. Revista Divulgação Saúde Debate, 18:43-50, 1997.

OLIVEIRA, M. L. C.; LOPES SILVA, M. T. A. Educação em Saúde: repensando a formação de professores. Revista Brasileira de Saúde Escolar, 1(2):3-20, 1990. ORGANIZAÇÃO MUNDIAL DA SAÚDE. Saúde do adolescente. Disponível em: http://www.who.int/maternal_child_adolescent/topics/adolescence/es/. Acesso em:

4 mar2012.

PELICIONI, M.C.F. As inter-relações entre educação, saúde e meio ambiente. Jornal da USP, São Paulo, v. 15, n. 494, p. 2, 08 nov., 1999.

PIAGET, J. Seis estudos de psicologia. Rio de Janeiro: Forense Universitária, 1998. PIRES, E.A.G.; DUARTE, M.F.S.; PIRES, M.C.; SOUZA, G.S. Hábitos de atividade física e o estresse em adolescentes de Florianópolis-SC, Brasil. Revista Brasileira de Ciência e Movimento, v. 12, n. 1, p. 51-6, 2004.

PITANGA, F.J.G. Epidemiologia, atividade física e saúde. Revista Brasileira de Ciência e Movimento, v. 10, n. 3, p.49-54, 2002.

PRIORE, S.E. Composição corporal e hábitos alimentares de adolescentes: uma contribuição à interpretação de indicadores de estado nutricional. São Paulo, 1998. (Tese de Doutorado - Universidade Federal de São Paulo/Escola Paulista de Medicina), 1998.

REIS, E. Estatística Descritiva. 4ª Ed. Lisboa: Silabo, 1998. 
ROLIM, M.K.S.B.; MATIAS, T.S.; SEGATO, L.; ANDRADE, A. Saúde percebida de adolescentes ativos e sedentários. Revista de Educação Física/UEM, Maringá, v. 18, p. 367-370, maio 2007.

SAMDAL, O.; NUTBEAM, D.; WOLD, B.; KANNAS, L. Achieving health and educational goals through schools - A study of the importance of the school climate and the students' satisfation with school. Health Education Research, v. 13, n. 3, p. 383397, 1998.

Sguizzatto GT, Garcez-Leme LE, Casimiro L. Evaluation of the quality of life among elderly female athletes. São Paulo Med J. 124(5):304-5, 2006. Disponível em:

<http://dx.doi.org/10.1590/S1516-31802006000500014>. Acesso em: 15 out2012. TRIVIÑOS, A.N.S. Introdução à pesquisa em ciências sociais: a pesquisa qualitativa em educação. São Paulo: Atlas, 1994.

VIEIRA, V.C.; PRIORE, S.E.; FISBERG, M. Atividade física na adolescência.

Adolesc. Latinoam. [online]. ago. 2002, v.3, n.1, p.0-0. Disponível em: <http://raladolec.bvs.br/scielo.php?script $=$ sci_arttext\&pid=S1414-

71302002000100007\&lng=pt\&nrm=iso>. ISSN 1414-7130. Acesso em 15abr2012. 